\title{
Editorial
}

\section{A yeast BH3-only protein for the rescue}

\author{
A Gross ${ }^{\star 1}$ \\ Cell Death and Differentiation (2011) 18, 1543-1544; doi:10.1038/cdd.2011.99
}

Mitochondrial outer membrane permeabilization (MOMP) is one of the most important processes that occur during apoptosis. In this process, the outer mitochondrial membrane is disrupted resulting in the release of proteins from the intermembrane space into the cytosol and activation of caspase proteases. How does MOMP occur? What are the proteins that are involved in its regulation? In addition, MOMP is part of a larger program termed the 'mitochondrial apoptotic program' that includes loss of the mitochondrial membrane potential $(\Delta \Psi \mathrm{m})$, an increase in the levels of reactive oxygen species (ROS), and more. How is the loss of $\Delta \Psi \mathrm{m}$ and increase in ROS involved in MOMP regulation? Many laboratories have been studying these questions for several decades now and nonetheless, this program remains largely a mystery.

The BCL-2 family members are major regulators of MOMP. ${ }^{1}$ The BH3-only pro-apoptotic proteins such as BID and BIM have a pivotal role in sensing and transducing apoptotic signals to the mitochondria. Once activated, these proteins translocate to the mitochondria to interact/activate the multi-domain pro-apoptotic proteins BAX and BAK, which in turn trigger MOMP. On the other hand, the anti-apoptotic BCL-2 family members such as BCL-2 and BCL- $X_{L}$ function as MOMP inhibitors by interacting with the activated proapoptotic members. BAX and BAK have a critical role in MOMP, as BAX and BAK double knockout cells are resistant to death-signal-induced MOMP and apoptosis. However, it remains unclear how these two proteins execute MOMP and whether there are additional proteins, especially resident mitochondrial proteins that have an active and critical role in this process.

Lower eukaryotes such as the yeast Saccahromyces cerevisiae are ideal systems to search for answers to complex processes such as MOMP. ${ }^{2} S$. cerevisiae is especially attractive, as members of the BCL-2 family were not identified yet in this organism, and thus can be used as a cellular system to 'reconstruct' MOMP from scratch. On the other hand, the lack of MOMP may suggest that the additional putative regulators of the process do not exist in yeast. Over the years, many research teams went 'fishing' in yeast for clues with relatively little success.

In a recent study published in EMBO Journal, the groups of Madeo and Kroemer report the identification of a novel

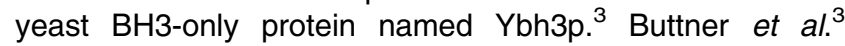
demonstrate that overexpression of $Y b h 3 p$ sensitizes the yeast $S$. cerevisiae to a variety of lethal insults, whereas its deletion protects yeast from these insults and prolongs replicative and chronological lifespan. Importantly, Ybh3p mediates cell death in a BH3-domain dependent manner as removing its putative $\mathrm{BH} 3$ domain eliminated death induction. Next, the authors characterized the nature of cell death and found that on lethal insults, Ybh3p translocates and integrates into the mitochondria membrane leading to cytochrome $c$ release, increase in ROS and loss of $\Delta \Psi \mathrm{m}$ (see Figure 1). Remarkably Ybh3p was found to interact with human BCL- $X_{L}$, and co-expression of BCL- $X_{L}$ suppressed the lethal effects of Ybh3p. Thus, Ybh3p seems to function like a classical mammalian $\mathrm{BH} 3-o n l y$ protein that is potent in inducing a mitochondrial apoptotic-like program in yeast.

Next Ybh3p was used as bait in an immunoperecipitation screen, and several mitochondrial resident proteins were identified. Among these proteins, Cor1p, a core subunit of the ubiqinol-cytochrome $c$ oxidoreductase complex, and Mir1p, a mitochondrial phosphate carrier, were found to be indispensable for Ybh3p-induced loss of $\Delta \Psi \mathrm{m}$ and cell death. Deletion of either Cor1p or Mir1p abolished stress-induced mitochondrial targeting of $\mathrm{Ybh} 3 \mathrm{p}$, arguing that both proteins contribute to Ybh3p-induced death by facilitating its translocation to mitochondria. Moreover, the protective effect of $\mathrm{YBH} 3$ deletion was completely abrogated in cells devoid of Mir1p, suggesting that Ybh3p functions via Mir1p to kill cells.

What is the relevance of these findings to apoptosis in higher eukaryotes? Interestingly, PHC/SLC25A3, the human ortholog of Mir1p, has been previously implicated as a regulator of apoptosis in human cells. ${ }^{4,5}$ Strikingly, Buttner et al. ${ }^{3}$ found that knockdown of either PHC or QCR1 (the human ortholog of Cor1p) in human osteosarcoma U2OS cells significantly reduced the mitochondrial targeting of BAX following treatment with the anti-cancer agent mitoxantrone. Moreover, knockdown of either PHC or QCR1 reduced mitoxantrone-induced loss of $\Delta \Psi \mathrm{m}$ and caspase activation. Thus, the studies in yeast seemed to have unraveled a phylogenetically conserved mechanism important for the targeting and activation of pro-apoptotic BCL-2 family proteins at the mitochondria (see Figure 1).

What next? One of the questions that arise from the Buttner et al. $^{3}$ studies is whether PHC and/or QCR1 function as receptors for selected BCL-2 family members such as BAX. This seems unlikely as both proteins are localized to the inner

\footnotetext{
1Department of Biological Regulation, The Weizmann Institute of Science, Rehovot 76100, Israel ${ }^{*}$ Corresponding author: A Gross, Department of Biological Regulation, The Weizmann Institute of Science, Rehovot 76100, Israel. Tel: + 972-8-9343656; Fax: +972-8-9344116; E-mail: atan.gross@weizmann.ac.il
} 


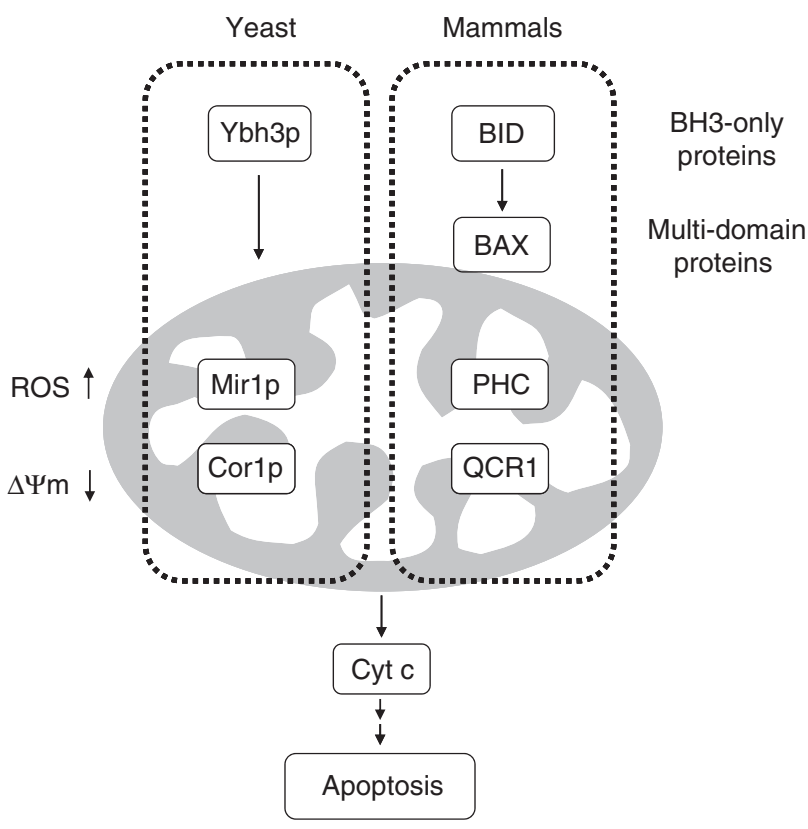

Figure 1 Similarities in the mitochondria cell death pathways of the yeast $S$. cerevisiae and of mammals. In yeast, the BH3-only protein Ybh3p translocates to the mitochondria and Mir1p and Cor1p are involved in its mitochondrial targeting leading to cytochrome $c$ (Cyt $c$ ) release, loss of $\Delta \Psi \mathrm{m}$ and increase in ROS levels. Similarly, in mammals, the $\mathrm{BH} 3$-only protein $\mathrm{BID}$ translocates to the mitochondria to activate BAX, which in turn relies on either PHC or QCR1 for its mitochondrial targeting leading to loss of $\Delta \Psi \mathrm{m}$ and Cyt $c$ release/caspase activation

mitochondrial membrane, and all BCL-2 family members are thought to be localized only to the outer membrane. Moreover, Ybh3p is localized to the outer membrane whereas its interactors Mir1p and Cor1p are localized to the inner membrane. Of note, it was recently demonstrated that mitochondrial carrier homolog 2 (MTCH2), a protein related to the mitochondrial carrier protein family and thus expected to localize to the inner membrane, is localized to the outer membrane and functions as a tBID receptor required for Fasinduced liver apoptosis. ${ }^{6}$ Thus, it is possible that PHC/Mir1p and/or QCR1/Cor1p also localize to the outer membrane. The other, perhaps more likely, possibility is that their activity in the inner membrane indirectly facilitates the mitochondrial targeting of selected BCL-2 proteins such as Ybh3p and BAX. This later possibility is extremely attractive as it suggests that the metabolic state of the mitochondria (which may affect the lipid composition of the outer membrane) may affect the susceptibility to apoptotic inducers. Another question that arises is whether the interaction of BCL-2 family members with resident mitochondrial proteins such as the one between Ybh3p and either Mir1p or Cor1p affects the activity of the later ones (e.g., phosphate transport in the case of Mir1p)? If this is indeed the case then perhaps modulating the activity of these proteins also contributes to MOMP regulation.

Does the yeast $S$. cerevisiae hold additional 'hidden jewels' that can help us in better understanding MOMP regulation? One of the powers of yeast is genetic screens, and Ybh3p can be used to screen for mutations that will either suppress or enhance its lethal effects. The studies recently published by Buttner et al. ${ }^{3}$ suggest that yeast have not yet said their last word and may reappear in the future with new 'rescues' to the MOMP puzzle.

\section{Conflict of Interest}

The author declares no conflict of interest.

Acknowledgements. Research in my laboratory is supported in part by the Israel Science Foundation, USA-Israel Binational Science Foundation (BSF), German-Israel Foundation (GIF), German-Israel Research Program in Cancer Research (IMOS-DKFZ), Israel Cancer Association, Minerva Stiftung and MDM ICR Research Award. AG is the incumbent of the Armour Family Career Development Chair of Cancer Research.

1. Youle RJ, Strasser A. Nat Rev Mol Cell Biol 2008; 9: 47-59.

2. Khoury CM, Greenwood MT. Biochim Biophys Acta 2008; 1783: 1449-1465.

3. Buttner S et al. EMBO J 2011 (in press).

4. Alcala $S$ et al. Oncogene 2008; 27 : 44-54

5. Poncet D et al. J Cell Biol 2006; 174: 985-996.

6. Zaltsman Y et al. Nat Cell Biol 2010; 12: 553-562. 